

\title{
SIZE-DEPENDENT FREE VIBRATION ANALYSIS OF INFINITE NANOTUBES USING ELASTICITY THEORY
}

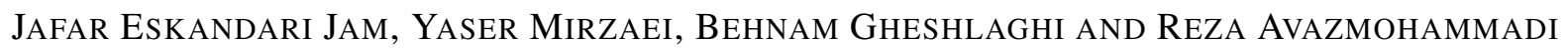

\begin{abstract}
Exact elasticity theory is employed to study the (two-dimensional) free vibration of nanoscale cylindrical tubes in the presence of free surface energy. Use is made of the Gurtin-Murdoch surface elasticity model to incorporate the surface stress terms into the pertinent boundary conditions. Some numerical examples are provided to depict the influence of the surface energy, and particularly the inner radius size of the nanocylinder, on the natural frequencies of the system. The results indicate a stronger influence of surface effects for both smaller values of the outer to inner radius ratio and higher modes of vibration.
\end{abstract}

\section{Introduction}

Due to accelerated miniaturization of components and devices in micro and nanoelectromechanical systems, there is an increasing demand for understanding of the behaviors of small-sized materials and structures. Nanostructured devices and materials have become progressively more important both in fundamental and applied research because of their unique physical properties [Tan and Lim 2006]. When the characteristic size of materials and devices shrinks to microns or nanometers, surface and interface effects start to play a considerable or even dominant role in their deformability, performance, and reliability, owing to the increasing ratio of surface/interface area to volume [Wong et al. 1997; Cuenot et al. 2004]. Also, atomic simulations have revealed that a solid surface/interface may be either elastically softer or stiffer than its bulk counterparts [Benveniste and Miloh 2001; Zhou and Huang 2004].

A continuum model of surface elasticity was first established in [Gurtin and Murdoch 1975] to account for the effects of surfaces, and further extended in [Gurtin et al. 1998] to incorporate the effects of interfaces as well. Surface elastic constants can be obtained through atomic calculations [Shenoy 2005]; their direct measurement has been very difficult until now. Investigations of the deformation of some elementary nanosized devices (for example, beams, tubes, and plates) have demonstrated that the predictions of Gurtin's surface/interface elasticity theory agree reasonably well with direct atomic simulations [Miller and Shenoy 2000; Shenoy 2002].

Although surface elasticity theory has been extensively used to elucidate the effects of various sizedependent phenomena on elastic fields in nanowires caused by static loadings [Chen et al. 2006; Jing et al. 2006], to date, however, investigation of surface effects on the dynamic behaviors of long nanotubes/nanowires has been comparatively lacking. As a few examples in the context of dynamic loadings in conjunction with surface elasticity theory, we mention studies regarding the diffraction of plane compressional/shear waves by nanosized inhomogeneities/voids (embedded in an elastic medium) [Wang 2007; Hasheminejad and Avazmohammadi 2009] which demonstrate the considerable importance of the

Yaser Mirzaei is the corresponding author.

Keywords: free surface energy, free vibration, nanotube, elasticity solution. 
surface effects on dynamic stress concentration around the nanoinhomogeneities. He and Lilley [2008] studied the influence of the surface stress on the resonance frequencies of bending nanowires using EulerBernoulli beam theory and, similarly, the natural frequencies of a microbeam in the presence of surface effects were estimated in [Abbasion et al. 2009] based on Timoshenko beam theory. Furthermore, in [Hasheminejad and Gheshlaghi 2010] a dissipative surface stress model was adopted to study the effect of size-dependent surface dissipation on natural frequencies of vibrating elastic nanowires. Nevertheless, as far as the authors know, investigation of surface effects on the natural frequencies of nanosized cylindrical tubes is still lacking. Here, we make use of classical elasticity theory in conjunction with Gurtin-Murdoch theory to carry out a (exact) two-dimensional natural frequency analysis of long, nanosized cylindrical tubes (nanotubes) in presence of free surface-energy effects.

\section{Formulation}

The elastic material of the nanotube (NT) under consideration is assumed to be linear, macroscopically homogeneous, and isotropic. Its constitutive equation may be written as

$$
\sigma_{i j}=\lambda \delta_{i j} \varepsilon_{j j}+2 \mu \varepsilon_{i j}
$$

where $\delta_{i j}$ is the Kronecker delta, $(\lambda, \mu)$ are the Lamé constants, and $\sigma_{i j}$ and $\varepsilon_{i j}$ are the stress and the strain tensors, respectively. The problem can be analyzed by means of the standard methods of elastodynamics. In the absence of body forces, the displacement field is governed by the classical Navier's equation [Pao and Mow 1973]

$$
\rho \frac{\partial^{2} \boldsymbol{u}}{\partial \boldsymbol{t}^{\mathbf{2}}}=\boldsymbol{\mu} \nabla^{2} \boldsymbol{u}+(\lambda+\mu) \nabla(\nabla \cdot \boldsymbol{u})
$$

subjected to appropriate boundary conditions. Here, $\rho$ is the elastic material density and $\boldsymbol{u}$ is the displacement vector that can advantageously be expressed as sum of the gradient of a scalar potential and the curl of a vector potential:

$$
\boldsymbol{u}=\nabla \varphi+\nabla \times \boldsymbol{\psi},
$$

with the condition $\boldsymbol{\nabla} \cdot \boldsymbol{\psi}=0$. The above decomposition enables us to separate the dynamic equation of motion (2) into the Helmholtz wave equations

$$
c_{p}^{2} \nabla^{2} \varphi=\ddot{\varphi}, \quad c_{s}^{2} \nabla^{2} \psi=\ddot{\psi},
$$

where $c_{p}^{2}=(\lambda+2 \mu) / \rho$ and $c_{s}^{2}=\mu / \rho$ are the propagation velocities of compressional and shear waves in the elastic medium, respectively, and superposed dots stand for the time derivative. Taking the divergencefree condition of $\psi$ into account, only two of the three components of $\psi$ remain independent. Also, considering the plane-strain assumption, (4) can be reduced to the following fully uncoupled scalar wave equations (see [Hasheminejad and Mirzaei 2009, Equations (b-2)]):

$$
c_{p}^{2} \nabla^{2} \phi=\ddot{\phi}, \quad c_{s}^{2} \nabla^{2} \psi=\ddot{\psi} .
$$

Furthermore, the relevant displacement components in polar coordinates, $(r, \theta)$, in terms of compressional and shear wave potentials may be simply written as [Pao and Mow 1973]

$$
u_{r}=\frac{\partial \phi}{\partial r}+\frac{1}{r} \frac{\partial \psi}{\partial \theta}, \quad u_{\theta}=\frac{1}{r} \frac{\partial \phi}{\partial \theta}-\frac{\partial \psi}{\partial r} .
$$


Correspondingly, the relevant stress components are

$$
\sigma_{r r}=\lambda \nabla^{2} \varphi+2 \mu\left[\frac{\partial^{2} \varphi}{\partial r^{2}}+\frac{\partial}{\partial r}\left(\frac{1}{r} \frac{\partial \psi}{\partial \theta}\right)\right], \quad \sigma_{r \theta}=\mu\left\{2 \frac{\partial}{\partial r}\left(\frac{1}{r} \frac{\partial \varphi}{\partial \theta}\right)+\frac{1}{r^{2}} \frac{\partial^{2} \psi}{\partial \theta^{2}}-r \frac{\partial}{\partial r}\left(\frac{1}{r} \frac{\partial \psi}{\partial r}\right)\right\} .
$$

Consider a long cylindrical NT with inner and outer radii $a$ and $b$, respectively. The in-plane cross section of the NT is shown in Figure 1. The field expansions for the standing compressional and shear waves within the tube (that is, the solutions to the wave equations (5)) with respect to the polar coordinate system may be written as [Pao and Mow 1973]

$$
\varphi(r, \theta, \omega)=\sum_{n=0}^{\infty}\left[a_{n} J_{n}(\alpha r)+b_{n} Y_{n}(\alpha r)\right] e^{i n \theta}, \quad \psi(r, \theta, \omega)=\sum_{n=0}^{\infty}\left[c_{n} J_{n}(\beta r)+d_{n} Y_{n}(\beta r)\right] e^{i n \theta},
$$

where $i=\sqrt{-1}, a_{n}$ through $d_{n}$ are unknown modal coefficients, $\alpha=\omega / c_{p}$ and $\beta=\omega / c_{s}$ are the compressional and shear wave numbers, respectively, $J_{n}$ and $Y_{n}$ are the cylindrical Bessel functions of the first and second kind, respectively, and $\omega$ is the circular frequency. Substituting (8) into (7), the relevant stress components can be respectively written as

$$
\begin{aligned}
& \sigma_{r r}(r, \theta, \omega)=\sum_{n=0}^{\infty}\left(a_{n} T_{1 n}^{(1)}+b_{n} T_{1 n}^{(2)}+c_{n} T_{2 n}^{(1)}+d_{n} T_{2 n}^{(2)}\right) e^{i n \theta}, \\
& \sigma_{r \theta}(r, \theta, \omega)=\sum_{n=0}^{\infty}\left(a_{n} T_{3 n}^{(1)}+b_{n} T_{3 n}^{(2)}+c_{n} T_{4 n}^{(1)}+d_{n} T_{4 n}^{(2)}\right) e^{i n \theta},
\end{aligned}
$$

in which

$$
\begin{aligned}
& T_{1 n}^{(i)}(r, \omega)=-2 \mu \frac{\alpha}{r} \ell_{n-1}^{(i)}(\alpha r)+\left[2 \mu \frac{n(1+n)}{r^{2}}-(\lambda+2 \mu) \alpha^{2}\right] \ell_{n}^{(i)}(\alpha r), \\
& T_{2 n}^{(i)}(r, \omega)=2 i \mu n\left[\frac{\beta}{r} \ell_{n-1}^{(i)}(\beta r)-\frac{(1+n)}{r^{2}} \ell_{n}^{(i)}(\beta r)\right], \\
& T_{3 n}^{(i)}(r, \omega)=2 i \mu n\left[\frac{\alpha}{r} \ell_{n-1}^{(i)}(\alpha r)-\frac{(1+n)}{r^{2}} \ell_{n}^{(i)}(\alpha r)\right], \\
& T_{4 n}^{(i)}(r, \omega)=2 \mu \frac{\beta}{r} \ell_{n-1}^{(i)}(\beta r)+\mu\left[-2 \frac{n(1+n)}{r^{2}}+\beta^{2}\right] \ell_{n}^{(i)}(\beta r),
\end{aligned}
$$

where $i=1,2$ and

$$
\ell_{n}^{(i)}= \begin{cases}J_{n} & (i=1) \\ Y_{n} & (i=2)\end{cases}
$$

According to surface elasticity theory, a surface is considered as a negligibly thin layer adhered to an abutting bulk material without slipping [Gurtin and Murdoch 1975; Gurtin et al. 1998]. The equilibrium and constitutive equations in the abutting (bulk) solids are the same as those in classical elasticity theory. However, the surface has different elastic constants than the solids. Hence, in general, a nonzero surface stress associated with the (nonzero) surface constants should be taken into account in order to derive the (localized) equilibrium equations on the surface. Here, for the sake of brevity, we only provide the final set of pertinent nonclassical boundary conditions (caused by presence of the surface stress) in the polar 


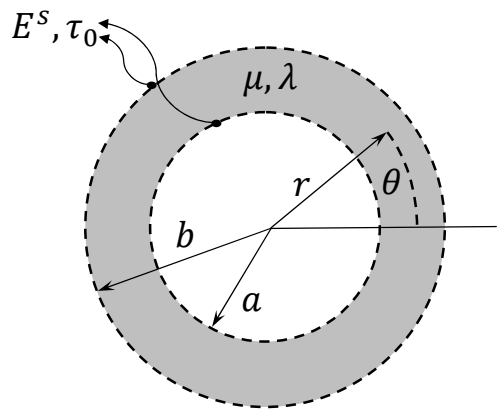

Figure 1. Problem geometry: two-dimensional view of the NT.

coordinate system [Wang et al. 2006]:

$$
\begin{array}{ll}
\sigma_{r r}(r=a, \theta, \omega)=\frac{1}{a} \sigma_{\theta \theta}^{(s)}(a, \theta, \omega), & \sigma_{r \theta}(r=a, \theta, \omega)=-\frac{1}{a} \frac{\partial \sigma_{\theta \theta}^{(s)}(a, \theta, \omega)}{\partial \theta}, \\
\sigma_{r r}(r=b, \theta, \omega)=-\frac{1}{b} \sigma_{\theta \theta}^{(s)}(b, \theta, \omega), & \sigma_{r \theta}(r=b, \theta, \omega)=\frac{1}{b} \frac{\partial \sigma_{\theta \theta}^{(s)}(b, \theta, \omega)}{\partial \theta},
\end{array}
$$

where $\sigma_{\theta \theta}^{(s)}(r, \theta, \omega)$ is the resultant surface stress at the surface with radius $r$ and is written as

$$
\sigma_{\theta \theta}^{(s)}(a, \theta, \omega)=\tau_{0}+E^{s} \varepsilon_{\theta \theta}(r=a, \theta, \omega), \quad \sigma_{\theta \theta}^{(s)}(b, \theta, \omega)=\tau_{0}+E^{s} \varepsilon_{\theta \theta}(r=b, \theta, \omega),
$$

in which $E^{s}$ is the (Young's modulus-type) elastic constant of the surface, $\varepsilon_{\theta \theta}=\left(\partial u_{\theta} / \partial \theta\right) / r$ and $\tau_{0}$ is the surface residual stress whose effect is not taken into account here (that is, we assume $\tau_{0}=0$ ). Making use of (6) and (8), the strain component $\varepsilon_{\theta \theta}$ may be written as

$$
\varepsilon_{\theta \theta}(r, \theta, \omega)=\sum_{n=0}^{\infty}\left(a_{n} T_{5 n}^{(1)}+b_{n} T_{5 n}^{(2)}+c_{n} T_{6 n}^{(1)}+d_{n} T_{6 n}^{(2)}\right) e^{i n \theta},
$$

where

$$
T_{5 n}^{(i)}(r, \omega)=r \alpha \ell_{n-1}^{(i)}(\alpha r)-n(1+n) \ell_{n}^{(i)}(\alpha r), \quad T_{6 n}^{(i)}(r, \omega)=i n\left(-r \beta \ell_{n-1}^{(i)}(\beta r)+(1+n) \ell_{n}^{(i)}(\beta r)\right) .
$$

Substitution of (9) and (12), along with (13), into the boundary conditions (11a) and (11b) leads to the following system of linear algebraic equations:

$$
\begin{aligned}
& \begin{aligned}
& a_{n}\left(T_{1 n}^{(1)}(a, \omega)-\right.\left.E^{s} T_{5 n}^{(1)}(a, \omega) / a\right)+b_{n}\left(T_{1 n}^{(2)}(a, \omega)-E^{s} T_{5 n}^{(2)}(a, \omega) / a\right) \\
&+c_{n}\left(T_{2 n}^{(1)}(a, \omega)-E^{s} T_{6 n}^{(1)}(a, \omega) / a\right)+d_{n}\left(T_{2 n}^{(2)}(a, \omega)-E^{s} T_{6 n}^{(2)}(a, \omega) / a\right)=0, \\
& a_{n}\left(T_{3 n}^{(1)}(a, \omega)+\right.\left.i n E^{s} T_{5 n}^{(1)}(a, \omega) / a\right)+b_{n}\left(T_{3 n}^{(2)}(a, \omega)+i n E^{s} T_{5 n}^{(2)}(a, \omega) / a\right) \\
&+c_{n}\left(T_{4 n}^{(1)}(a, \omega)+i n E^{s} T_{6 n}^{(1)}(a, \omega) / a\right)+d_{n}\left(T_{4 n}^{(2)}(a, \omega)+i n E^{s} T_{6 n}^{(2)}(a, \omega) / a\right)=0 \\
& a_{n}\left(T_{1 n}^{(1)}(b, \omega)+E^{s} T_{5 n}^{(1)}(b, \omega) / b\right)+b_{n}\left(T_{1 n}^{(2)}(b, \omega)+E^{s} T_{5 n}^{(2)}(b, \omega) / b\right) \\
& \quad+c_{n}\left(T_{2 n}^{(1)}(b, \omega)+E^{s} T_{6 n}^{(1)}(b, \omega) / b\right)+d_{n}\left(T_{2 n}^{(2)}(b, \omega)+E^{s} T_{6 n}^{(2)}(b, \omega) / b\right)=0,
\end{aligned}
\end{aligned}
$$




$$
\begin{aligned}
a_{n}\left(T_{3 n}^{(1)}(b, \omega)-\right. & \left.i n E^{s} T_{5 n}^{(1)}(b, \omega) / b\right)+b_{n}\left(T_{3 n}^{(2)}(b, \omega)-i n E^{s} T_{5 n}^{(2)}(b, \omega) / b\right) \\
& +c_{n}\left(T_{4 n}^{(1)}(b, \omega)-i n E^{s} T_{6 n}^{(1)}(b, \omega) / b\right)+d_{n}\left(T_{4 n}^{(2)}(b, \omega)-i n E^{s} T_{6 n}^{(2)}(b, \omega) / b\right)=0 .
\end{aligned}
$$

This system can be cast in the form

$$
\boldsymbol{T}_{n} \boldsymbol{d}_{n}=\mathbf{0}
$$

in which $\boldsymbol{d}_{n}=\left[a_{n}, b_{n}, c_{n}, d_{n}\right]^{T}(n=0,1,2, \ldots)$ is the modal vector multiplied by $(4 \times 4)$ square matrix $\boldsymbol{T}_{n}$, containing frequency-dependent coefficients. Setting the determinant of $\boldsymbol{T}_{n}$ equal to zero, the characteristic equation of the system is obtained which leads to determination of the natural frequencies. It should be noted that for each frequency number, $n$, there are infinite numbers of longitudinal modes, denoted by $m$. A Mathematica program was written for numerical calculation of the natural frequencies as a function of the NT inner and outer radii through a simple root finding technique based on the bisection approach.

\section{Numerical examples}

To illustrate the influence of the surface stress on the dynamic behavior of the nanotube, some numerical examples are provided in this section. To this end, a NT with an infinite length and of selected outer to inner radius ratio is considered to be made of isotropic aluminum with the following physical properties: $\rho=2700 \mathrm{~kg} / \mathrm{m}^{3}, \lambda=52.0 \times 10^{9} \mathrm{~N} / \mathrm{m}^{2}$, and $\mu=34.7 \times 10^{9} \mathrm{~N} / \mathrm{m}^{2}$. Two different sets of surface properties corresponding to the crystallographic directions [100] (denoted as surface A (SA)) and [111] (denoted as surface B (SB)) in aluminum are used in the calculation. The corresponding elastic constants are [Hasheminejad and Avazmohammadi 2009] $E^{s}=-8.95 \mathrm{~N} / \mathrm{m}$ for SA and $E^{s}=6.08 \mathrm{~N} / \mathrm{m}$ for SB. Surface C (SC) (with $E^{s}=0 \mathrm{~N} / \mathrm{m}$ ) refers to a surface with the classical perfect bonding condition. Furthermore, as mentioned before, we assume $\tau_{0}=0$. In the following examples, the natural frequencies have been normalized by $c_{p} / b$.

Figure 2 displays the variation of the normalized natural frequency versus the inner radius of the NT, for the first longitudinal mode $(m=1)$ and for two selected outer to inner radius ratios $(b / a=1.1,1.5)$. These figures include the results associated with the first two frequencies numbers $(n=0,1)$ for three different surface types, SA, SB, and SC. Some observations are in order. It can be seen that the surface effect is more evident for lower values of the outer to inner radius ratio, corresponding to thinner NTs. Also, the frequency values for SA and SB are, respectively, lower and higher than that of the classical SC. This drop (rise) for SA (SB) is connected to the negative (positive) sign of the associated constant $E^{s}$. Moreover, since the absolute value of $E^{s}$ for SA is greater than that of SB, its discrepancy with the classic solution (SC) is larger. Furthermore, as expected, by increasing the inner radius of NT (specifically, for $a>15 \mathrm{~nm}$ ), the surface effect gradually diminishes and the normalized frequencies (associated with SA and SB) approach the classical (size-independent) limit (we used [Gazis 1958, Equation (17)] to obtain the classical solution).

Figure 3 displays the variation of the normalized natural frequency, for three types of surfaces, SA, $\mathrm{SB}$, and SC, versus the frequency number $(1 \leq n \leq 8)$ for a NT with $b / a=3$. The results are calculated for the first three longitudinal modes (that is, $1 \leq m \leq 3$ ) at each frequency number, $n$. It is found that the surface effects become more evident as the frequency number, $n$, increases. Similarly, a larger surface effect is observed at higher longitudinal mode numbers. This may be linked to the fact that the deformed 

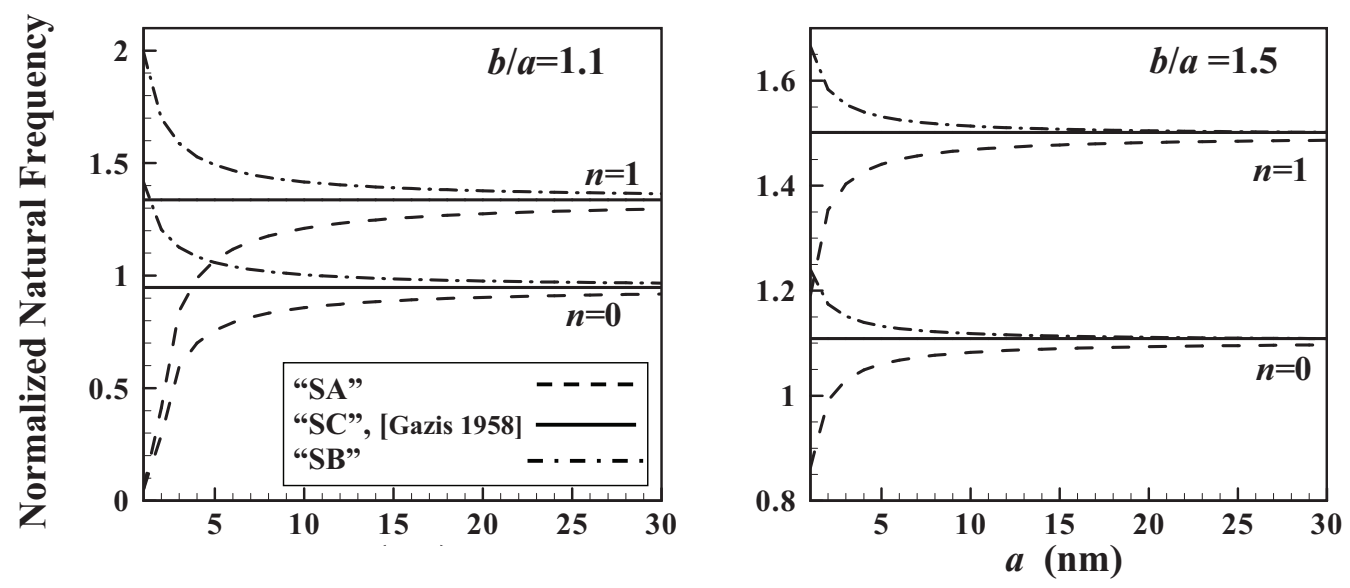

Figure 2. Variation of the normalized natural frequency with NT size, for selected radius ratio, surface type, longitudinal mode $(m=1)$, and frequency number $(n=0,1)$.

shape of the inner/outer surfaces of the NT at higher modes of vibration has more curvature associated with a larger hoop strain at the surfaces. Hence, based on (12), a stronger surface effect at these modes is anticipated. Also, the fundamental frequency number $(n=2)$ that has the minimum value among all frequencies remains unchanged despite the presence of the surface effect.

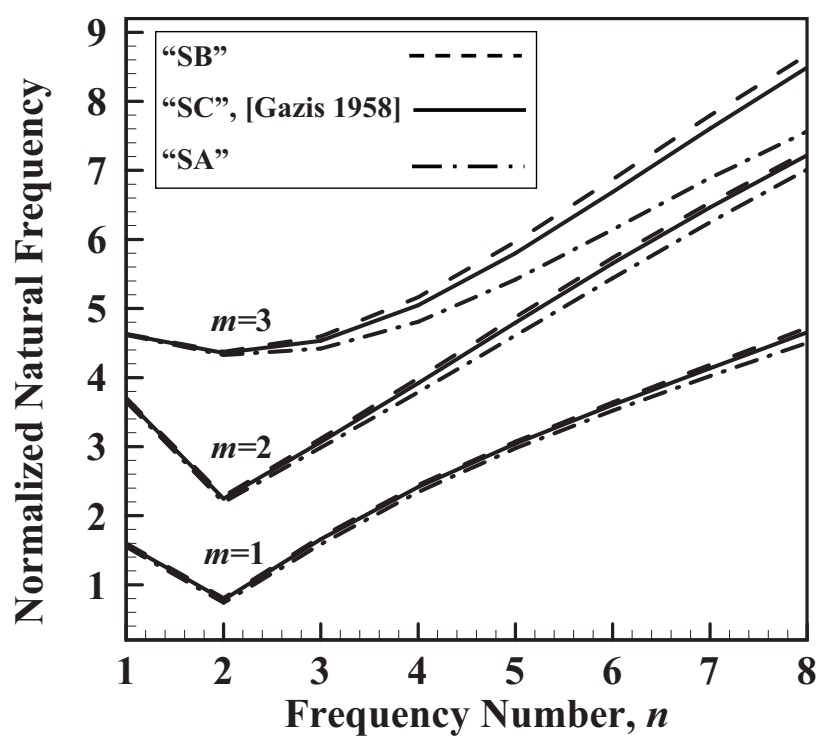

Figure 3. Variation of the normalized natural frequency with frequency number $(n)$, for selected surface types and longitudinal modes $(m=1,2,3)$. 


\section{Conclusions}

Making use of the Helmholtz wave equations together with the theory of surface elasticity, the influence of surface stress was investigated on the natural frequencies of a long, nanoscaled, cylindrical tube with a circular cross-section. As a numerical example, the free vibration of a nanotube (NT) made of aluminum with two different types of surface constants was studied. In this example, the size-dependence of the natural frequencies of the NT was analyzed for various natural frequency numbers and longitudinal modes. It was found that the size dependence is more noticeable at higher modes of vibration. This observation could be considerable technological interest in the area of designing nanoscaled devices.

\section{References}

[Abbasion et al. 2009] S. Abbasion, A. Rafsanjani, R. Avazmohammadi, and A. Farshidianfar, "Free vibration of microscaled Timoshenko beams", Appl. Phys. Lett. 95:14 (2009), 143122.

[Benveniste and Miloh 2001] Y. Benveniste and T. Miloh, "Imperfect soft and stiff interfaces in two-dimensional elasticity", Mech. Mater. 33:6 (2001), 309-323.

[Chen et al. 2006] C. Q. Chen, Y. Shi, Y. S. Zhang, J. Zhu, and Y. J. Yan, "Size dependence of Young's modulus in ZnO nanowires", Phys. Rev. Lett. $96: 7$ (2006), 75505.

[Cuenot et al. 2004] S. Cuenot, C. Frétigny, S. Demoustier-Champagne, and B. Nysten, "Surface tension effect on the mechanical properties of nanomaterials measured by atomic force microscopy", Phys. Rev. B 69:16 (2004), 165410.

[Gazis 1958] D. C. Gazis, "Exact analysis of the plane-strain vibrations of thick-walled hollow cylinders", J. Acoust. Soc. Am. 30:8 (1958), 786-794.

[Gurtin and Murdoch 1975] M. E. Gurtin and A. I. Murdoch, "A continuum theory of elastic material surfaces", Arch. Ration. Mech. Anal. 57:4 (1975), 291-323.

[Gurtin et al. 1998] M. E. Gurtin, J. Weissüuller, and F. Larché, "A general theory of curved deformable interfaces in solids at equilibrium", Philos. Mag. A 78:5 (1998), 1093-1109.

[Hasheminejad and Avazmohammadi 2009] S. M. Hasheminejad and R. Avazmohammadi, "Size-dependent effective dynamic properties of unidirectional nanocomposites with interface energy effects", Compos. Sci. Technol. 69:15-16 (2009), 25382546.

[Hasheminejad and Gheshlaghi 2010] S. M. Hasheminejad and B. Gheshlaghi, "Dissipative surface stress effects on free vibrations of nanowires", Appl. Phys. Lett. 97:25 (2010), 253103.

[Hasheminejad and Mirzaei 2009] S. M. Hasheminejad and Y. Mirzaei, "Free vibration analysis of an eccentric hollow cylinder using exact 3D elasticity theory”, J. Sound Vib. 326:3-5 (2009), 687-702.

[He and Lilley 2008] J. He and C. M. Lilley, "Surface stress effect on bending resonance of nanowires with different boundary conditions", Appl. Phys. Lett. 93:26 (2008), 263108.

[Jing et al. 2006] G. Y. Jing, H. L. Duan, X. M. Sun, Z. S. Zhang, J. Xu, Y. D. Li, J. X. Wang, and D. P. Yu, "Surface effects on elastic properties of silver nanowires: contact atomic-force microscopy", Phys. Rev. B 73:23 (2006), 235409.

[Miller and Shenoy 2000] R. E. Miller and V. B. Shenoy, "Size-dependent elastic properties of nanosized structural elements", Nanotechnology 11:3 (2000), 139-147.

[Pao and Mow 1973] Y. H. Pao and C. C. Mow, Diffraction of elastic waves and dynamics stress concentration, Crane Russak, New York, 1973.

[Shenoy 2002] V. B. Shenoy, "Size-dependent rigidities of nanosized torsional elements", Int. J. Solids Struct. 39:15 (2002), 4039-4052.

[Shenoy 2005] V. B. Shenoy, "Atomistic calculations of elastic properties of metallic fcc crystal surfaces", Phys. Rev. B 71:9 (2005), 094104.

[Tan and Lim 2006] E. P. S. Tan and C. T. Lim, "Mechanical characterization of nanofibers: a review", Compos. Sci. Technol. 66:9 (2006), 1102-1111. 
[Wang 2007] G. F. Wang, "Diffraction of plane compressional wave by a nanosized spherical cavity with surface effects", Appl. Phys. Lett. 90:21 (2007), 211907.

[Wang et al. 2006] G. F. Wang, T. J. Wang, and X. Q. Feng, "Surface effects on the diffraction of plane compressional waves by a nanosized circular hole", Appl. Phys. Lett. 89:23 (2006), 231923.

[Wong et al. 1997] E. W. Wong, P. E. Sheehan, and C. M. Lieber, "Nanobeam mechanics: elasticity, strength, and toughness of nanorods and nanotubes", Science 277:5334 (1997), 1971-1975.

[Zhou and Huang 2004] L. G. Zhou and H. Huang, “Are surfaces elastically softer or stiffer?”, Appl. Phys. Lett. 84:11 (2004), 1940-1942.

Received 28 Mar 2011. Revised 8 Aug 2011. Accepted 19 Aug 2011.

JAFAR ESKANDARI JAM: jejam@mail.com

Composite Material and Technology Center, Tehran, Iran

YASER MIRZAEI: mirzaei@damavandiau.ac.ir

Department of Mechanical Engineering, Damavand Branch, Islamic Azad University, Damavand, Iran

BEHNAM GHESHLAGHI: behnamqeshlaqi@gmail.com

Young Researchers Club, Science and Research Branch, Islamic Azad University, Tehran, Iran

REZA AVAZMoHAMmadi: reza.avazmohammadi@gmail.com

Department of Mechanical Engineering and Applied Mechanics, University of Pennsylvania, 220 S 33rd St.,

Philadelphia, PA 19104-6315, United States 


\title{
JOURNAL OF MECHANICS OF MATERIALS AND STRUCTURES
}

\author{
jomms.net
}

\author{
Founded by Charles R. Steele and Marie-Louise Steele \\ EDITORS \\ Charles R. STEele \\ DAVIDE BIGONI \\ Stanford University, USA \\ University of Trento, Italy \\ IWONA JASIUK University of Illinois at Urbana-Champaign, USA \\ YASUHIDE SHINDO Tohoku University, Japan
}

\section{EDITORIAL BOARD}

H. D. Bui École Polytechnique, France

J. P. CARTER University of Sydney, Australia

R. M. CHRISTENSEN Stanford University, USA

G. M. L. GLadWELL University of Waterloo, Canada

D. H. Hodges Georgia Institute of Technology, USA

J. HUTCHINSON Harvard University, USA

C. HwU National Cheng Kung University, Taiwan

B. L. KARIHALOO University of Wales, UK

Y. Y. KIM Seoul National University, Republic of Korea

Z. Mroz Academy of Science, Poland

D. PAmplona Universidade Católica do Rio de Janeiro, Brazil

M. B. RUBIN Technion, Haifa, Israel

A. N. SHUPIKOV Ukrainian Academy of Sciences, Ukraine

T. TARNAI University Budapest, Hungary

F. Y. M. WAN University of California, Irvine, USA

P. WRIGGERS Universität Hannover, Germany

W. YANG Tsinghua University, China

F. ZIEGLER Technische Universität Wien, Austria

PRODUCTIONｃontact@msp.org

Silvio LEVY Scientific Editor

See http://jomms.net for submission guidelines.

JoMMS (ISSN 1559-3959) is published in 10 issues a year. The subscription price for 2012 is US $\$ 555 /$ year for the electronic version, and \$735/year (+\$60 shipping outside the US) for print and electronic. Subscriptions, requests for back issues, and changes of address should be sent to Mathematical Sciences Publishers, Department of Mathematics, University of California, Berkeley, CA 94720-3840.

JoMMS peer-review and production is managed by EditFLOw ${ }^{\circledR}$ from Mathematical Sciences Publishers.

\author{
mathematical sciences publishers \\ http://msp.org/ \\ A NON-PROFIT CORPORATION \\ Typeset in $\mathrm{IAT}_{\mathrm{E}} \mathrm{X}$ \\ Copyright $@ 2012$ by Mathematical Sciences Publishers
}




\title{
Journal of Mechanics of Materials and Structures
}

\author{
Volume 7, No.2 February 2012
}

Micromechanical analysis of unidirectional composites using a least-squares-based differential quadrature element method

Mohammad Bayat and Mohammad Mohammadi Aghdam

Size-dependent free vibration analysis of infinite nanotubes using elasticity theory

JAFAR ESKandari JaM, YASER Mirzaei, Behnam GHeShlaghi

and ReZa AvaZmohammadi

Spectral element model for the vibration of a spinning Timoshenko shaft

USIK LEE and INJOON JANG

On indenter boundary effects at elastic contact

Denis Jelagin and Per-Lennart LaRsson 165

Reflection of $P$ and $S V$ waves from the free surface of a two-temperature thermoelastic solid half-space

BALJEET SingH and KirAN BALA

A nonlinear Timoshenko beam formulation based on strain gradient theory ReZa Ansari, RAHEb GHOLAmi and MOHAMMAD ALI DARABi

Finite element analysis of bending-stiff composite conical shells with multiple delamination 